\title{
INVESTIGATION ON THE EFFECTS OF HEDGE HORIZON AND ESTIMATION PERIOD ON HEDGING EFFECTIVENESS: AN APPLICATION ON TURKISH FUTURES MARKETS
}

DOI: 10.17261/Pressacademia.2021.1513

PAP- V.14-2021(39)-p.143-147

\section{Sevgi Eren}

İstanbul Commerce University, Graduate School of Finance, Istanbul, Turkey. seren@ticaret.edu.tr ,ORCID: 0000-0003-1495-5302

\section{To cite this document}

Eren, S. (2021). Investigation on the effects of hedge horizon and estimation period on hedging effectiveness: an application on Turkish futures markets. PressAcademia Procedia (PAP), 14, 143-147.

Permanent link to this document: http://doi.org/10.17261/Pressacademia.2021.1513

Copyright: Published by PressAcademia and limited licensed re-use rights only.

\begin{abstract}
Purpose- Risk management in financial markets is possible with the effective use of hedging in futures markets. This study was carried out to examine the impact of different hedging horizons and different estimation periods on the hedge ratio and hedge efficiency of the porftolios.

Methodology- In the study, Rolling Regression (Moving Window Procedure) method was used to obtain the hedge ratio and measure the hedge effectiveness. Spot and futures market data for the period 2010-2021 were used in the analyses. Within the framework of ex-post hedging and ex-ante hedging, the findings regarding the hedge ratio and hedging effectiveness were evaluated separately, and the reliability of the findings was tested. It has been investigated how hedging performed with different hedging horizon and different estimation periods performs on portfolios.

Bulgular-In the study, it was found that the hedge ratio and hedge efficiency tended to increase for the majority of portfolios as the hedging horizon and estimation period increased, and it was concluded that the results obtained with the hypothesis tests carried out were statistically significant. In addition, the hedging performances were also investigated and it was determined that the increase in the hedging horizon and estimation period provide a higher reduction of the portfolio risk.

Results: The results of the study are largely in line with the literature although they differ in terms of different hedging horizons, estimation periods and portfolios for ex-post and ex-ante hedging. Hedging horizon and estimation periods have a significat impact on hedge ratio and hedging effectiveness. The other crucial result of the study is that the performance of the rolling regression model is good as the most common used methods, GARCH Models, in estimation of the hedge ratio.
\end{abstract}

Keywords: Hedging, hedge ratio, hedge effectiveness, hedging horizon, estimation period JEL Codes: G11, G32, P34

\section{KORUNMA ZAMANININ VE TAHMIN PERIYODUNUN KORUNMA ETKINLIĞi ÜZERINE OLAN ETKISININ INCELENMESI: TÜRKIYE VADELI IŞLEM PIYASALARI ÜZERINE BIR UYGULAMA}

\section{ÖZET}

Amaç- Riski yönetmede vadeli işlem piyasalarını kullanan yatırımcılar için spot piyasada sahip oldukları pozisyon karşıı̆ı̆nda vadeli işlem piyasasında nasıl bir pozisyon almaları gerektiği ve edinmeleri gereken sözleşme sayısını iyi analiz edebilmek önem kazanmıştır. Bu anlamda sözleşme sayısını belirlemek amacıyla kullanılan hedge oranını etkin şekilde tahminleyebilmek, risk yönetimi amacıyla futures piyasalarda işlem yapan piyasa aktörleri için büyük öneme sahip olmaktadır. Bu çalışma, farklı korunma zamanları ve farklı tahmin periyotlarının hedge oranı ve hedge etkinliğine olan etkisinin incelenmesi amacıyla gerçekleştirilmiştir.

Yöntem- Çalışmada hedge oranı elde etmede ve hedge etkinliğini ölçmede dinamik regresyon (rolling regression) metodu kullanılmıştır. Analizler, 2010-2021 dönemi spot ve vadeli işlem piyasa verileri kullanılarak ex-post hedging ve ex-ante hedging çerçevesinde gerçekleştirilmiş ve farklı tahmin periyotları ve korunma zamanları ele alınarak bu bağlamda incelenmiştir. Elde edilen bulgular, hipotez testlerinden t-testi yardımıyla güvenilirlik açısından sınanmıştır. Ardından ise hedge performansı analiz edilerek gerçekleştirilen hedging sonrasında riskte meydana gelen değişim incelenmiştir. Bu bağlamda ex-post ve ex-ante hedging için bulgular ayrı ayrı değerlendirilmiş, elde edilen bulguların güvenilirliği test edilerek farklı korunma zamanları ve farklı tahmin periyotlarıyla gerçekleştirilen hedging işleminin portföyler üzerinde nasıl bir performans sergilediği araştırılmıştır. 
Bulgular- Çalışma sonuçlarına göre ex-post ve ex-ante hedging için farklı korunma zamanları, tahmin periyotları ve portföyler özelinde farklılıklar gösterse de literatürle büyük oranda paralellik taşımaktadır. Çalışmada korunma zamanı ve tahmin periyodu arttıkça hedge oranının ve hedge etkinliğinin portföylerin çoğunluğu için artma eğiliminde olduğu tespit edilmiş, gerçekleştirilen hipotez testleriyle de elde edilen sonuçların büyük oranda anlamlılık gösterdiği sonucuna ulaşılmıştır. Bununla birlikte gerçekleştirilen hedging performansları da incelenmiş olup korunma zamanı ve tahmin periyodu artışının portföy riskinin daha yüksek oranda düşürülmesine katkı sağladığı tespit edilmiştir.

Sonuç- Çalışma sonuçlarına göre korunma zamanı ve tahmin periyodunun hedge oranı ve hedge etkinliği üzerinde etkili olduğu tespit edilmiştir. Uygulanan dinamik regresyon modelinin de literatürde kullanılan ve daha yeni ve güncel modellerden olan GARCH modelleri kadar iyi performans sergileyebileceği çalışma sonucunda ortaya konmuştur.

Anahtar Kelimeler: Hedging, Hedge Oranı, Hedge Etkinliği, Korunma Zamanı, Tahmin Periyodu JEL Kodları: G11, G32, P34

\section{REFERENCES}

Avcı, E., Çinko, M., \& Çinko, L. (2009). Hisse Senedi Portföylerinde Riskten Korunma. Maliye Finans Yazıları, 85, 27-37.

Aydın, Z. (2007). İşletmelerde Döviz Futures Sözleşmeler ile Kur Riski Yönetimi: VOB (Vadeli İşlemler ve Opsiyon Borsası) Uygulaması. Yayımlanmamış Yüksek Lisans Tezi. Adnan Menderes Üniversitesi Sosyal Bilimler Enstitüsü: Aydın.

Barbi, M., \& Romagnoli, S. (2014). A Copula-Based Quantile Risk Measure Approach to Estimate the Optimal Hedge Ratio. The Journal of Futures Markets, 34(7), 658-675.

Başçı, E. S. (2003). Vadeli işlem Piyasası Aracı Olarak Swap'ın İşleyişi ve Finansal Piyasalardaki Kullanımları. Gazi Üniversitesi Endüstriyel Sanatlar Eğitim Fakültesi Dergisi, 11(12), 18-33.

Bera, A. K., Garcia, P., \& Roh, J.-S. (1997). Estimation of Time-Varying Hedge Ratios for Corn and Soybeans: Bgarch and Random. The Indian Journal of Statistics, 59(3), 346-368.

Bekaert, G., Harvey, C. R. (1997). Emerging Equity Market Volatility. Journal of Financial Economics, 43, 29-77.

Bollerslev, T. (1986). Generalized Autoregressive Conditional Heteroskedasticity. Journal of Econometrics, 31, 307-327.

Borsa İstanbul. (2017). Sorularla Vadeli İşlemler ve Opsiyon Borsası. ViOP.

Borsa İstanbul. (2021). 2020 Entegre Faaliyet Raporu. Borsa İstanbul. İstanbul

Borsa İstanbul. VIOP Tanıtım Kitapçığı Opsiyon Sözleşmeleri. İstanbul: Borsa İstanbul Vadeli İşlem ve Opsiyon Piyasası.

Butterworth, D., Holmes, P. (2000). Ex-Ante Hedging Effectiveness of UK Stock Index Futures Contracts: Evidence for the FTSE 100 and FTSE Mid 250 Contracts. European Financial Management, 6(4), 441-457.

Büberkökü, Ö. (2019). Bist 30 Endeksi ve Dolar-TL Kuru için Futures Kontratlara Dayalı Optimal Hedge Rasyolarının ve Hedging Etkinliğinin İncelenmesi: Kapsamlı Bir Analiz. Finans Ekonomi ve Sosyal Araştırmalar Dergisi, 4(4), 515-544.

Çelik, İ. (2012). Vadeli İşlem Piyasasında Fiyat Keşfi İzmir Vadeli İşlem ve Opsiyon Borsasında Ampirik Bir Uygulama. İstanbul: Türkiye Bankalar Birliği, Elma Basım.

Çelik, İ. (2014). Vadeli İşlem Piyasasında Optimal Hedge Rasyosunun Statik ve Dinamik Teknikler Yardımıyla Hesaplanması. Uluslararası Alanya İşletme Fakültesi Dergisi, 6(3), 1-13.

Chen, R., \& vd. (2020). Futures minimum variance hedge ratio determination: An ex-ante analysis. North American Journal of Economics, 54, $1-17$.

Chen, S. S., Lee, C. F., \& Shrestha, K. (2004). An Empirical Analysis of the Relationship Between the Hedge Ratio and Hedging Horizon: A Simultaneous Estimation of the Short- and Long-Run Hedge Ratios. The Journal of Futures Markets, 24(4), 359-386.

Choudhry, T. (2003). Short-run deviations and optimal hedge ratio: evidence from stock futures. Journal of Multinational Financial Management, 13, 171-192.

Choudhry, T. (2009). Short-run deviations and time-varying hedge ratios: Evidence from agricultural futures markets. International Review of Financial Analysis, 18, 58-65.

Çiftçi, Ö. H. (2011). Türev Piyasalar ve Türk Bankacılık Sektöründeki Uygulamalar. Yayınlanmamış Yüksek Lisans Tezi. Süleyman Demirel Üniversitesi Sosyal Bilimler Enstitüsü: Isparta.

Conlon, T., \& Cotter, J. (2012). An Empirical Analysis of Dynamic Multiscale Hedging Using Wavelet Decomposition. The Journal of Futures Markets, 32(3), 272-299. 
Degiannakis, S., \& Floros, C. (2010). Hedge Ratios in South African Stock Index Futures. Journal of Emerging Market Finance, 9(3), $285-304$. Demirtaş, Ö., \& Güngör, Z. (2004). Portföy Yönetimi ve Portföy Seçimine Yönelik Uygulama. Havacılık ve Uzay Teknolojileri Dergisi, 1(4), 103109.

Ederington, L. H. (1979). The Hedging Performance of the New Futures Markets. The Journal of Finance, 34(1), 157-170.

Elton, E. J., Gruber, M. J. (1977). Risk Reduction and Portfolio Size: An Analytical Solution, The Journal of Business, 50(4), $415-437$.

Emhan, A. (2009). Risk Yönetim Süreci ve Risk Yönetmekte Kullanılan Teknikler. Atatürk Üniversitesi İktisadi ve İdari Bilimler Dergisi, 23(3), 209-220.

Engle, R. F., Granger, C. W. J. (1987). Co-Integration and Error Correction: Representation, Estimation and Testing. Econometrica, 55(2), 251276.

Er, H., Ateş, A. (2015). The Hedging Effectiveness and the Stability of the Optimal Hedge Ratios: Evidence for the Istanbul Exchange 30 Contract. Journal of Business, Economics and Finance, 4(3), 351-362.

Ersoy, E. (2011). Spot ve Vadeli İşlem Piyasaları Arasındaki Fiyat ve Volatilite İlişkisi: iMKB-VOB Örneği. Yayımlanmamış Doktora Tezi. Erciyes Üniversitesi Sosyal Bilimler Enstitüsü: Kayseri.

Evci, S., \& Kandır, S. Y. (2017). Optimal Hedge Oranı Tahmini: Dolar/TL vadeli İşlem Sözleşmeleri Üzerine Bir Uygulama. TisK Akademi, 186200.

Fabozzi, F. J., Modigliani, F., \& Jones, F. J. (2010). Foundationsd of Financial Markets and Institutions. Boston: Pearson Education, Inc.

Fan, R., Li, H., \& Park, S. Y. (2016). Estimation and Hedging Effectiveness of Time-Varying Hedge Ratio: Nonparametric Approaches. The Journal of Futures Markets, 36(10), 968-991.

Figlewski, S. (1985). Hedging with Stock Index Futures: Theory and Application in a New Market. The Journal of Futures Markets, 5(2), 183199.

Floros, C., \& Vougas, D. V. (2006). Hedging Effectiveness in Greek Stock Index Futures Market, 1999-2001. International Research Journal of Finance and Economics, 5, 7-18.

Gök, I. Y. (2016). Türkiye Pay Endeks Futures Piyasasında Optimum Korunma Oranı ve Korunma Etkililiği. Ege Akademik Bakış, 16(4), 719-732.

Gözgör, G. (2008). Finansal Türev Piyasaları: Forward, Futures, Opsiyon ve Döviz Üzerine Bir Uygulama. Yayımlanmamış Yüksek Lisans Tezi. İstanbul Üniversitesi Sosyal Bilimler Enstitüsü: İstanbul.

Gümrah, Ü., \& Gökbulut, R. İ. (2017). Hedging Performance of Turkish Stock Index Futures. Balkan Journal of Social Sciences, 6(12), $133-141$. Hatemi-J, A., \& El-Katib, Y. (2012). Stochastic optimal hedge ratio: theory and evidence. Applied Economics Letters, 19, $699-703$.

Holmes, P. (1996). Stock Index Futures Hedging: Hedge Ratio Estimation, Duration Effects, Expiration Effects and Hedge Ratio Stability. Journal of Business Finance \& Accounting, 23(1), 63-77.

Horcher, K. A. (2005). Essentials of Financial Risk Management. New Jersey: John Wiley \& Sons, Inc.

Hull, J. C. (2015). Options, Futures and Other Derivatives (9 ed.). Toronto: Pearson.

In, F., \& Kim, S. (2006). The Hedge Ratio and the Empirical Relationship between the Stock and Futures Markets: A New Approach Using Wavelet Analysis. The Journal of Business, 79(2), 799-820.

Johnson, L. L. (1960). The Theory of Hedging and Speculation in Commodity Futures. The Review of Economic Studies, 27(3), 139-151.

Juhl, T., Kawaller, I. G., \& Koch, P. D. (2012). The Effect of the Hedge Horizon on Optimal Hedge Size and Effectiveness When Prices are Cointegrated. The Journal of Futures Markets, 32(9), 937-976.

Karan, M. B. (2018). Yatırım Analizi ve Portföy Yönetimi. Ankara: Gazi Kitabevi.

Karatepe, Y. (2000). Türev Piyasaları. Ankara: Ankara Üniversitesi Siyasi Bilgiler Fakültesi Yayını.

Kavussanos, M. G., \& Visvikis, I. (2008). Hedging effectiveness of the Athens stock index futures contracts. European Journal of Finance, 14(3), 243-270.

Kayalıdere, K., Aracı, H., \& Aktaş, H. (2012). Türev Ve Spot Piyasalar Arasındaki Etkileşim: VOB Üzerine Bir İnceleme. Muhasebe ve Finansman Dergisi, 137-154.

Köroğlu, Y. (2019). Finansal Risk Yönetiminde Karma Dağılım Modeli. Yayımlanmamış Yüksek Lisans Tezi. Necmettin Erbakan Üniversitesi Fen Bilimleri Enstitüsü: Konya.

Kostika, E., \& Markellos, R. N. (2013). Optimal Hedge Ratio Estimation and Effectiveness Using ARCD. Journal of Forecasting, 32, 41-50. 
Kütük, Ö. (2014). Türev Araçlar ve Türk Bankacılık Sektöründeki Uygulamaları. Yayımlanmamış Yüksek Lisans Tezi. Başkent Üniversitesi Sosyal Bilimler Enstitüsü: Ankara.

Lai, Y. (2018). Estimation of the optimal futures hedge ratio for equity index portfolios using a realized beta generalized autoregressive conditional heteroskedasticity model. Journal of Futures Market, 38, 1370-1390.

Lee, C., Lin, F., \& Chen, M. (2010). International Hedge Ratios for Index Futures Market: A Simultaneous Equations Approach. Review of Pacific Basin Financial Markets and Policies, 13(2), 203-213.

Lee, C., Wang, K., \& Chen, Y. L. (2009). Hedging and Optimal Hedge Ratios for International Index Futures Markets. Review of Pacific Basin Financial Markets and Policies, 12(4), 593-610.

Lien, D., \& Shrestha, K. (2007). An Empirical Analysis of the Relationship Between Hedge Ratio and Hedging Horizon Using Wavelet Analysis. The Journal of Futures Markets, 27(2), 127-150.

Lien, D., Shrestha, K., \& Wu, J. (2016). Quantile Estimation of Optimal Hedge Ratio. The Journal of Futures Markets, 36(2), $194-2014$.

Lien, D., Tse, Y., \& Tsui, A. K. (2002). Evaluating the hedging performance of the constant-correlation GARCH model. Applied Financial Economics, 12(11), 791-798.

Lindahl, M. (1992). Minimum Variance Hedge Ratios for Stock Index Futures: Duration and Expiration Effects. The Journal of Futures Markets, 12(1), 33-53.

Liu, W. (2014). Optimal hedge ratio estimation and hedge effectiveness with multivariate skew distributions. Applied Economics, 46(12), $1420-1435$

Malliaris, A. G., Urrutia, J. L. (1991). The Impact of the Lengths of Estimation Periods and Hedging Horizons on the Effectiveness of a Hedge: Evidence from Foreign Currency Futures. The Journals of Futures Markets, 11(3), 271-289.

Marcopoulou, C. E. (2016). Realized hedge ratio: Predictability and hedging performance. International Review of Financial Analysis, 45, 121133.

MSCI. (2021). MSCI Turkey Index (EUR) Report. MSCI.

Odabaşı, S. (2014). Endeks Vadeli İşlem Sözleşmeleri ile Spot Piyasa Arasındaki Etkileşimlerin İrdelenmesine Yönelik BisT30 Üzerinde Bir Çalışma. Yayımlanmamış Doktora Tezi. İstanbul Üniversitesi Sosyal Bilimler Enstitüsü: İstanbul.

Olgun, O., \& Yetkiner, I. H. (2011). Determination of Optimal Hedging Strategy for Index Futures: Evidence from Turkey. Emerging Markets Finance \& Trade, 47(6), 68-79.

Özaydın, O. (2018). Vadeli BIST 30 Endeksi Kontratları Üzerine Koruma Oranı Tahmini ve Koruma Oranı Etkinliği. Bankacılık ve Sermaye Piyasası Araştırmaları Dergisi-BSPAD, 2(6), 16-27.

Öznacar, R. (2006). Vadeli İşlemlerin Para Piyasalarına Etkileri. Yayımlanmamış Yüksek Lisans Tezi. İstanbul Üniversitesi Sosyal Bilimler Enstitüsü: İstanbul.

Saltoğlu, B. (2020). Türev Araçlar, Piyasalar ve Risk Yönetimi. Sermaye Piyasası Lisanslama Sicil ve Eğitim Kuruluşu Türev Araçlar Sınavı Çalışma Notları.

Salvador, A., \& Manzana, V. (2014). Measuring Hedging Effectiveness of Index Futures Contracts: Do Dynamic Models Outperform Static Models? A Regime Switching Approach. Journal of Futures Market, 34(4), 374-398.

Saraç, M. (2015). Finansal Yönetim. İstanbul: İstanbul Üniversitesi İktisat Fakültesi.

Satıcı, Ö. (2015). Opsiyonlar. Working Paper. Ankara Üniversitesi Sosyal Bilimler Enstitüsü: Ankara.

Sephton, P. S. (1993). Optimal Hedge Ratios at the Winnipeg Commodity Exchange. The Canadian Journal of Economics, 26(1), $175-193$.

SPDR MSCI Emerging Markets UCITS ETF. (2021). State Street Global Advisors.

Stoll, H. R., \& Whaley, R. E. (1993). Futures and Options: Theory and Applications. Ohio: South-Western Publishing Co.

Sultan, J., \& vd. (2019). Hedging performance of multiscale hedge ratios. Journal of Futures Markets, 39, $1613-1632$.

Tanrı̈ven, C., \& Aksoy, E. E. (2011). Sistematik Riskin Belirleyicileri: ĐMKB'de Sektörel Karsılastırma. Muhasebe ve Finansman Dergisi, 111138.

The National Association of Pension Funds Limited. (2013). Derivatives and Risk Management Made Simple. London: National Association of Pension Funds Limited.

TDK. (2021). Türk Dil Kurumu Sözlükleri. Erişim: https://sozluk.gov.tr/ 
Tekir, Y. C. (2019). Döviz Vadeli İşlem Sözleşmelerinde Riskten Korunma Oranının Hesaplanması. Yayımlanmamış Yüksek Lisans Tezi. Çukurova Üniversitesi Sosyal Bilimler Enstitüsü: Adana.

Tunalı, E. (2009). Vadeli İşlemler Piyasaları ve Türkiye Vadeli İşlem ve Opsiyon Borsası VOB ile Londra Finansal Futures ve Opsiyon Borsası LIFFE'nin Karşılaştırılması. Yayınlanmamış Yüksek Lisans Tezi. Trakya Üniversitesi/Sosyal Bilimler Enstitüsü: Edirne.

Tuncay, F. E., \& Cengiz, H. (2016). Faize Dayalı Swap Sözleşmeleri ve Muhasebeleştirilmesi. International Journal of Economic and Administrative Studies, 16, 1-22.

Weber E.J. (2009) A Short History of Derivative Security Markets. In: Hafner W., Zimmermann H. (eds) Vinzenz Bronzin's Option Pricing Models. Springer, Berlin, Heidelberg.

Yalçın, C. Ç. (2015). Türk Bankacılık Sektöründe Türev Ürünler ve Türev Ürünlerin Kullanımını Etkileyen Faktörlerin Analizi. Yayımlanmamış Yüksek Lisans Tezi. Gazi Üniversitesi Sosyal Bilimler Enstitüsü: Ankara.

Yang, W. (2000). M-GARCH Hedge Ratios And Hedging Effectiveness In Australian Futures Markets. Yayımlanmamış Yüksek Lisans Tezi. Edith Cowan University: Perth.

Yang, W., \& Allen, D. E. (2004). Multivariate GARCH hedge ratios and hedging effectiveness in Australian futures markets. Accounting and Finance, 45, 301-321.

Yücel, T., Mandacı, P. E., \& Kurt, G. (2007). İşletmelerin Finansal Yönetimi Risk Yönetimi ve Türev Ürün Kullanımı: İMKB 100 Endeksinde Yer Alan İşletmelerde Bir Uygulama. Muhasebe ve Finansman Dergisi(36), 1-9.

Zhou, J. (2016). Hedging performance of REIT index futures: A comparison of alternative hedge ratio estimation methods. Economic Modelling, 52, 690-698.

Zulkifli, M., Basarudin, S. B., Norzaidi, M. D., Siong, S.C. (2008). A study on diversification in Malaysian stock market, EABR \& TLC Conderence. 